\title{
Tail Behaviour of Weighted Sums of Order Statistics of Dependent Risks
}

\author{
Enkelejd Hashorva ${ }^{1}$ \& Jinzhu Li ${ }^{2}$
}

Abstract: Let $X_{1}, \ldots, X_{n}$ be $n$ real-valued dependent random variables. With motivation from Mitra and Resnick (2009), we derive the tail asymptotic expansion for the weighted sum of order statistics $X_{1: n} \leq \cdots \leq X_{n: n}$ of $X_{1}, \ldots, X_{n}$ under the general case in which the distribution function of $X_{n: n}$ is long-tailed or rapidly varying and $X_{1}, \ldots, X_{n}$ may not be comparable in terms of their tail probability. We also present two examples and an application of our results in risk theory.

Keywords: aggregated risk; Gumbel max-domain of attraction; long-tailed distribution; Mitra-Resnick conditions; weighted sums

Mathematics Subject Classification: Primary 62P05; Secondary 62E10, $91 \mathrm{~B} 30$

\section{Introduction}

In numerous finance, insurance and risk management applications, a prevailing model for the maximum and the total sum of dependent risks is the lognormal one; see, e.g., Foss and Richards (2010), Asmussen et al. (2011), Gulisashvili and Tankov (2013), Kortschak and Hashorva (2013) and Embrechts et al. (2014). The asymptotic tail behaviour of the total sum (or aggregated risk) of lognormal based models has been first derived in Asmussen and Rojas-Nandayapa (2008). A key characteristic of lognormal risks is that they are rapidly varying. By resorting to extreme value theory, Mitra and Resnick (2009) offered a new methodology for the investigation of the tail asymptotics of the total sum of rapidly varying risks. Given the fact that in applications risks are almost always dependent, the aforementioned paper constitutes a significant achievement in understanding the extremal behaviour of the maximum and the total sum of dependent risks. In particular, for dependent nonnegative random variables (risks) $X_{1}, \ldots, X_{n}$ such that $\lim _{x \rightarrow \infty} \mathbb{P}\left(X_{i}>x\right) / \mathbb{P}\left(X_{1}>x\right)=\lambda_{i} \in[0, \infty)$ for $1 \leq i \leq n$, under some weak dependence assumptions (referred to in this paper as Mitra-Resnick conditions), Mitra and Resnick (2009) showed that if $X_{1}$ has a distribution function in the Gumbel max-domain of attraction (see below for the definition), then

$$
\mathbb{P}\left(S_{n}>x\right) \sim \mathbb{P}\left(X_{n: n}>x\right) \sim\left(\sum_{i=1}^{n} \lambda_{i}\right) \mathbb{P}\left(X_{1}>x\right), \quad x \rightarrow \infty,
$$

where $S_{n}=\sum_{i=1}^{n} X_{i}$ is the aggregated risk, $X_{1: n} \leq \cdots \leq X_{n: n}$ are the order statistics of $X_{1}, \ldots, X_{n}$, and " $\sim$ " means that the ratio of the two sides converges to 1 .

The recent contribution Asimit et al. (2013) showed further that, for some positive weights $c_{0}, \ldots, c_{n-1}$, the weighted $\operatorname{sum} \sum_{i=0}^{n-1} c_{i} X_{n-i: n}$ has the following asymptotic behaviour:

$$
\mathbb{P}\left(\sum_{i=0}^{n-1} c_{i} X_{n-i: n}>x\right) \sim \mathbb{P}\left(c_{0} X_{n: n}>x\right) \sim\left(\sum_{i=1}^{n} \lambda_{i}\right) \mathbb{P}\left(c_{0} X_{1}>x\right), \quad x \rightarrow \infty,
$$

if the risks $X_{1}, \ldots, X_{n}$ obey the Mitra-Resnick conditions, or $X_{1}$ has a regularly varying distribution function and the risks are asymptotically independent.

However, we can not obtain the tail asymptotics of $S_{n}$ or $\sum_{i=0}^{n-1} c_{i} X_{n-i: n}$ by the methodology given in the aforementioned papers if there is no proportional tail-relationship among $X_{1}, \ldots, X_{n}$, i.e., $\lambda_{1}, \ldots, \lambda_{n}$ do

\footnotetext{
${ }^{1}$ Faculty of Business and Economics (HEC Lausanne), University of Lausanne, Lausanne, Switzerland

${ }^{2}$ School of Mathematical Science and LPMC, Nankai University, Tianjin 300071, P.R. China
} 
not exist. An interesting example where this is indeed the case is that of log-normal risks with random variances presented in Example 4.1 below.

The principal goal of this contribution is to adapt the Mitra-Resnick methodology for dependent risks which, in terms of their tail behaviour, may not be comparable. We shall deal with both the longtailed and rapidly varying (real-valued) random variables allowing for three broad dependence models. Roughly speaking, under our setup, we shall show in Theorem 3.1 below that

$$
\mathbb{P}\left(\sum_{i=0}^{n-1} c_{i} X_{n-i: n}>x\right) \sim \mathbb{P}\left(c_{0} X_{n: n}>x\right) \sim \sum_{i=1}^{n} \mathbb{P}\left(c_{0} X_{i}>x\right), \quad x \rightarrow \infty
$$

holds uniformly for $\left(c_{0}, \ldots, c_{n-1}\right)$ in some compact set.

The rest of this paper is organized as follows. Section 2 gives some definitions of asymptotic theory and some preliminary results. Our main results are presented in Section 3 followed by examples and an application in Section 4. The proofs of all the results are relegated to Section 5.

\section{Preliminaries}

Hereafter, all limit relations hold as $x \rightarrow \infty$ unless otherwise stated. As usual, for two positive functions $a(x)$ and $b(x)$, we write $a(x)=o(b(x))$ or $a(x)=o(1) b(x)$ if $\lim _{x \rightarrow \infty} a(x) / b(x)=0$. Moreover, a realvalued random variable is always assumed to be not only concentrated on $(-\infty, 0]$. For a real-valued random variable $X$ with distribution function $F$, we call $X$ or $F$ heavy-tailed if the corresponding moment generating function $\widehat{F}(\delta)=\int_{-\infty}^{\infty} \mathrm{e}^{\delta x} \mathrm{~d} F(x)$ diverges to $\infty$ for all $\delta>0$; otherwise we call $X$ or $F$ light-tailed.

Almost all commonly used heavy-tailed distributions belong to the long-tail class. By definition, a real-valued random variable $X$ with distribution function $F=1-\bar{F}$ is long-tailed, denoted by $X \in \mathcal{L}$ or $F \in \mathcal{L}$, if $\bar{F}(x)>0$ for any $x \geq 0$ and $\bar{F}(x+y) \sim \bar{F}(x)$ for any $y \in \mathbb{R}$; see, e.g., Foss et al. (2013). In this case, we can define two associated sets of eventually positive functions

$$
\mathcal{H}_{X}=\mathcal{H}_{F}=\{h(\cdot): h(\cdot) \text { satisfies (i)-(iii) }\}
$$

and

$$
\mathcal{H}_{X}^{*}=\mathcal{H}_{F}^{*}=\mathcal{H}_{F} \cap\{h(\cdot): h(x) \rightarrow \infty\},
$$

where properties (i)-(iii) are specified as:

(i) $h(x)=o(x)$;

(ii) $\bar{F}(x+y h(x)) \sim \bar{F}(x)$ for any $y \in \mathbb{R}$;

(iii) $h(\cdot)$ is weakly self-neglecting (introduced by Asmussen and Foss (2014)), i.e.,

$$
\limsup _{x \rightarrow \infty} \frac{h(x+y h(x))}{h(x)}<\infty, \quad \forall y \in \mathbb{R}
$$

Property (iii) is a weakened version of the concept of self-neglecting, which requires further that

(iii') $h(x+y h(x)) \sim h(x)$ for any $y \in \mathbb{R}$.

A positive function $l(\cdot)$ is slowly varying if $l(x y) \sim l(x)$ for any $y>0$. The set $\mathcal{H}_{F}^{*}$ is non-empty, since in view of Lemma 4.1 of $\mathrm{Li}$ et al. (2010) there exists some slowly varying function $h(\cdot)$ (naturally selfneglecting) such that $h(x) \rightarrow \infty$ and properties (i)-(ii) hold. Additionally, $\mathcal{H}_{F}^{*}$ may also contain nonslowly-varying functions. For instance, if $F$ is regularly varying, i.e., $\bar{F}(x) \sim l(x) x^{-\alpha} \in \mathcal{R}_{-\alpha}$ for some 
$\alpha \geq 0$ and some slowly varying function $l(\cdot)$, then one can easily check that $h(x)=x^{p}(1+\beta \sin x) \in \mathcal{H}_{F}^{*}$ for any $p \in(0,1)$ and $\beta \in(-1,1)$.

A real-valued random variable $X$ with distribution function $F$ having upper endpoint $x_{F}:=\sup \{x$ : $F(x)<1\}$ belongs to the Gumbel max-domain of attraction (GMDA) if there exists some positive scaling function $h(\cdot)$ such that

$$
\lim _{x \rightarrow x_{F}} \frac{\bar{F}(x+y h(x))}{\bar{F}(x)}=\mathrm{e}^{-y}, \quad \forall y \in \mathbb{R} .
$$

In this case we write $X \in \operatorname{GMDA}(h)$ or $F \in \operatorname{GMDA}(h)$.

Remark 2.1. Hereafter a scaling function $h(\cdot)$ of some distribution function belonging to the GMDA may not be the one specified in properties (i)-(iii) and (iii'). Hence, the $h(\cdot)$ in Assumption $\mathbf{A}$ is not necessarily related to the $h(\cdot)$ in Assumption $\mathbf{B}$ or $\mathbf{C}$ below. The unified symbol $h(\cdot)$ for such functions is used to simplify the writing of our assumptions and proofs below.

According to extreme value theory, the normalized maxima of a random sample with underlying distribution function in the GMDA converge in distribution to a Gumbel random variable. Additionally, if $F$ belongs to the GMDA with $x_{F}=\infty$, then it belongs to the class of rapid variation specified by the relation $\lim _{x \rightarrow \infty} \bar{F}(x y) / \bar{F}(x)=0$ for any $y>1$. Furthermore, if $F \in \operatorname{GMDA}(h)$ with $x_{F}=\infty$, then $h(\cdot)$ satisfies property (iii') mentioned above and $h(x)=o(x)$. See, e.g., Resnick (1987) or Embrechts et al. (1997) for these well-known results.

The class of univariate distributions in the GMDA includes both light-tailed and heavy-tailed distributions with exponential distributions and heavy-tailed Weibull distributions as respective examples. On the other hand, if $F \in \operatorname{GMDA}(h)$ and $h(x) \rightarrow \infty$ (implying $\left.x_{F}=\infty\right)$, then $F \in \operatorname{GMDA}(h) \cap \mathcal{L}$ and $h^{p}(\cdot) \in \mathcal{H}_{F}^{*}$ for any $p \in(0,1)$. Conversely, $F \in \operatorname{GMDA}(h) \cap \mathcal{L}$ implies $h(x) \rightarrow \infty$ by Lemma 2.1 of Goldie and Resnick (1988). Hence, summarizing the above analysis, we arrive at:

Lemma 2.1. $F \in \operatorname{GMDA}(h)$ and $h(x) \rightarrow \infty \Longleftrightarrow F \in \operatorname{GMDA}(h) \cap \mathcal{L} \Rightarrow h^{p}(\cdot) \in \mathcal{H}_{F}^{*}$ for any $p \in(0,1)$.

\section{Main Results}

Recall that $X_{1}, \ldots, X_{n}$ are $n$ real-valued dependent random variables and $X_{1: n} \leq \cdots \leq X_{n: n}$ are the corresponding order statistics. Enlightened by Assumptions 2.1-2.5 of Mitra and Resnick (2009), we shall consider in this paper the following dependence structure:

A. $X_{n: n} \in \operatorname{GMDA}(h)$ with a distribution function having an infinite upper endpoint. Further, it holds that

$$
\lim _{x \rightarrow \infty} \frac{\mathbb{P}\left(\left|X_{i}\right|>t h(x), X_{j}>x\right)}{\mathbb{P}\left(X_{n: n}>x\right)}=0 \text { for any } 1 \leq i \neq j \leq n \text { and any } t>0,
$$

and

$$
\lim _{x \rightarrow \infty} \frac{\mathbb{P}\left(X_{i}>L h(x), X_{j}>L h(x)\right)}{\mathbb{P}\left(X_{n: n}>x\right)}=0 \text { for any } 1 \leq i<j \leq n \text { and some } L>0 .
$$

We remark that the original Mitra-Resnick conditions include relations (3.1) and (3.2) with the maximum $X_{n: n}$ replaced by $X_{1}$. Clearly, utilizing the maximum $X_{n: n}$ instead of $X_{1}$ relaxes the constraint of our assumption. Additionally, compared with the original Mitra-Resnick conditions, we drop the nonnegativity of the risks and the tail-relationships among the risks. These improvements make our Assumption A more extensive and allow us to study some flexible dependence structures; see Examples 4.1 and 4.2 below for details. A drawback of Assumption A lies in that it is not easy to show 
$X_{n: n} \in \operatorname{GMDA}(h)$ in general. To overcome this drawback, we present Lemma 4.1 below, which gives a simple condition to verify $X_{n: n} \in \operatorname{GMDA}(h)$.

In addition to the dependence structure controlled by Assumption $\mathbf{A}$, we shall also investigate the asymptotic tail behaviour of weighted sums of order statistics of dependent risks under the following long-tail case:

B. $X_{n: n} \in \mathcal{L}$ and there exists some $h(\cdot) \in \mathcal{H}_{X_{n: n}}$ such that relations (3.1) and (3.2) hold.

A positive function $h(\cdot)$ is dominatedly varying if $0<\liminf _{x \rightarrow \infty} h(x y) / h(x) \leq \limsup _{x \rightarrow \infty} h(x y) / h(x)<$ $\infty$ for any $y>0$. For a real-valued random variable $X$ with distribution function $F$, we write $X \in \mathcal{D}$ or $F \in \mathcal{D}$ if $\bar{F}$ is dominatedly varying; see, e.g., Embrechts et al. (1997) and Cai and Tang (2004) for the details. The last case considered in this paper is as follows:

C. $X_{n: n} \in \mathcal{L} \cap \mathcal{D}$ and there exists some dominatedly varying $h(\cdot) \in \mathcal{H}_{X_{n: n}}$ such that relation (3.1) holds.

Now, we are ready to state our main theorem, which implies the max-sum equivalence of $X_{1}, \ldots, X_{n}$ when $c_{0}=c_{1}=\cdots=c_{n-1}=1$. As mentioned before, compared with Corollary 2.2 of Mitra and Resnick (2009), it contains the long-tail case and drops the nonnegativity of $X_{1}, \ldots, X_{n}$ and the tailrelationships among $X_{1}, \ldots, X_{n}$.

Theorem 3.1. Let $X_{1}, \ldots, X_{n}$ be $n$ real-valued random variables. If one of Assumptions $\mathbf{A}-\mathbf{C}$ holds, then for any $0<a \leq b<\infty$ and $0 \leq d<\infty$ relation (1.1) holds uniformly for $\left(c_{0}, c_{1}, \ldots, c_{n-1}\right) \in$ $[a, b] \times[0, d]^{n-1}$.

From the proof of Theorem 3.1 below, we have a corresponding result for nonnegative $X_{1}, \ldots, X_{n}$ with real-valued weights:

Corollary 3.1. In addition to the conditions of Theorem 3.1, if $X_{1}, \ldots, X_{n}$ are nonnegative, then (1.1) holds uniformly for $\left(c_{0}, c_{1}, \ldots, c_{n-1}\right) \in[a, b] \times[-d, d]^{n-1}$.

Based on Theorem 3.1 and Corollary 3.1, by conditioning on the values of $C_{0}, \ldots, C_{n-1}$, we obtain the following corollary, in which the assertion under Assumption $\mathbf{C}$ generalizes Theorem 1.1 of Yang (2014).

Corollary 3.2. Under the conditions of Theorem 3.1, let $C_{0}, \ldots, C_{n-1}$ be $n$ arbitrarily dependent random variables independent of $X_{1}, \ldots, X_{n}$ such that $\mathbb{P}\left(a \leq C_{0} \leq b\right)=\mathbb{P}\left(0 \leq C_{i} \leq d\right)=1$ for $1 \leq i \leq$ $n-1$. Then, we have

$$
\mathbb{P}\left(\sum_{i=0}^{n-1} C_{i} X_{n-i: n}>x\right) \sim \mathbb{P}\left(C_{0} X_{n: n}>x\right) \sim \sum_{i=1}^{n} \mathbb{P}\left(C_{0} X_{i}>x\right) .
$$

If further $X_{1}, \ldots, X_{n}$ are nonnegative, then (3.3) holds given that $\mathbb{P}\left(a \leq C_{0} \leq b\right)=\mathbb{P}\left(\left|C_{i}\right| \leq d\right)=1$ for $1 \leq i \leq n-1$.

For $n$ mutually independent random variables $X_{1}, \ldots, X_{n}$, it is of interest to seek conditions such that they are max-sum equivalent; see Embrechts and Goldie (1980), Cai and Tang (2004), Geluk (2009), Li and Tang (2010) and the references therein. This is connected to the well-known principle of a single big jump in risk theory; see, e.g., Embrechts et al. (1997) or Foss et al. (2007).

Next, we discuss Assumptions $\mathbf{A}-\mathbf{C}$ for independent $X_{1}, \ldots, X_{n}$. By Lemma 2.1 and Lemma 5.1(b) below, in the independence case our Assumptions $\mathbf{A}-\mathbf{C}$ have their respective counterparts as follows: 
$\mathbf{A}^{\prime} . X_{n: n} \in \operatorname{GMDA}(h) \cap \mathcal{L}$ and

$$
\lim _{x \rightarrow \infty} \frac{\mathbb{P}\left(X_{i}>L h(x)\right) \mathbb{P}\left(X_{j}>L h(x)\right)}{\mathbb{P}\left(X_{n: n}>x\right)}=0 \text { for any } 1 \leq i \neq j \leq n \text { and some } L>0 .
$$

$\mathbf{B}^{\prime} . X_{n: n} \in \mathcal{L}$ and there exists some $h(\cdot) \in \mathcal{H}_{X_{n: n}}^{*}$ such that relation $\left(3.2^{\prime}\right)$ holds.

$\mathbf{C}^{\prime} . X_{n: n} \in \mathcal{L} \cap \mathcal{D}$.

Hence, as mentioned before, our Theorem 3.1 indicates the max-sum equivalence of independent random variables meeting one of Assumptions $\mathbf{A}^{\prime}-\mathbf{C}^{\prime}$. However, the assertion under Assumption $\mathbf{C}^{\prime}$ is covered by a more general existing result presented in Theorem 1 of $\mathrm{Li}$ and Tang (2010). We conclude the assertions under Assumptions $\mathbf{A}^{\prime}$ and $\mathbf{B}^{\prime}$ by the following corollary.

Corollary 3.3. Let $X_{1}, \ldots, X_{n}$ be $n$ real-valued and mutually independent random variables with $X_{n: n} \in \mathcal{L}$. If either (a) $X_{n: n} \in \operatorname{GMDA}(h) \cap \mathcal{L}$ and (3.2') holds or (b) there exists some $h(\cdot) \in \mathcal{H}_{X_{n: n}}^{*}$ such that (3.2') holds, then $X_{1}, \ldots, X_{n}$ are max-sum equivalent, i.e.,

$$
\mathbb{P}\left(\sum_{i=1}^{n} X_{i}>x\right) \sim \sum_{i=1}^{n} \mathbb{P}\left(X_{i}>x\right) .
$$

\section{Examples and Application}

We begin this section with a fundamental lemma, which will be applied in the verification of the examples given below. Actually, this lemma provides a way to verify that $X_{n: n}$ belongs to some distribution class considered in this paper.

Lemma 4.1. Let $X_{1}, \ldots, X_{n}$ be $n$ real-valued random variables. Assume that

$$
\lim _{x \rightarrow \infty} \frac{\mathbb{P}\left(X_{i}>x, X_{j}>x\right)}{\mathbb{P}\left(X_{n: n}>x\right)}=0 \text { for any } 1 \leq i<j \leq n .
$$

(a) If $X_{i} \in \operatorname{GMDA}\left(h_{i}\right)$ with $h_{i}(x) \rightarrow \infty$ and $h_{i}(x) \sim h_{1}(x)$ for every $1 \leq i \leq n$, then $X_{n: n} \in$ $\operatorname{GMDA}\left(h_{1}\right)$.

(b) If $X_{i} \in \mathcal{L}$ or $\mathcal{D}$ or $\mathcal{R}_{-\alpha}$ (for some $\alpha>0$ ) for every $1 \leq i \leq n$, then $X_{n: n} \in \mathcal{L}$ or $\mathcal{D}$ or $\mathcal{R}_{-\alpha}$, respectively.

Proof. Note the fact that

$$
\sum_{i=1}^{n} \mathbb{P}\left(X_{i}>x\right)-\sum_{1 \leq i<j \leq n} \mathbb{P}\left(X_{i}>x, X_{j}>x\right) \leq \mathbb{P}\left(X_{n: n}>x\right) \leq \sum_{i=1}^{n} \mathbb{P}\left(X_{i}>x\right) .
$$

Hence, relation (4.1) implies that

$$
\mathbb{P}\left(X_{n: n}>x\right) \sim \sum_{i=1}^{n} \mathbb{P}\left(X_{i}>x\right) .
$$

In view of Resnick (1987), relation (2.1) holds locally uniformly for $y \in \mathbb{R}$. Hence, for assertion (a), using relation (4.3) and $X_{i} \in \operatorname{GMDA}\left(h_{i}\right)$ with $h_{i}(x) \sim h_{1}(x)$ for every $1 \leq i \leq n$, it is easy to obtain relation (2.1) with $h(x)$ replaced by $h_{1}(x)$ for the tail probability of $X_{n: n}$. Assertion (b) immediately follows from relation (4.3) and the definitions of the classes $\mathcal{L}, \mathcal{D}$, and $\mathcal{R}_{-\alpha}$. 
Next, we present two examples satisfying Assumption $\mathbf{A}$ and Assumptions $\mathbf{B}-\mathbf{C}$, respectively. In both examples there is no necessary tail-relationship among the random variables $X_{1}, \ldots, X_{n}$.

Example 4.1. Let $\left(Y_{1}, \ldots, Y_{n}\right)$ be a multivariate normal random vector with mean vector $\left(\mu_{1}, \ldots, \mu_{n}\right)$ and covariance matrix $\left(\rho_{i j} \sigma_{i} \sigma_{j}\right)_{n \times n}$, where $\sigma_{i}>0, \rho_{i i}=1$ for $1 \leq i \leq n$ and $-1<\rho_{i j}=\rho_{j i}<1$ for $1 \leq i \neq j \leq n$. Let further $W_{1}, \ldots, W_{n}$, independent of $\left(Y_{1}, \ldots, Y_{n}\right)$, be $n$ nonnegative and arbitrarily dependent random variables with finite and positive upper endpoints. Now we verify Assumption $\mathbf{A}$ for the random variables $X_{1}=\mathrm{e}^{W_{1} Y_{1}}, \ldots, X_{n}=\mathrm{e}^{W_{n} Y_{n}}$.

Since $\mu_{i}$ and $\sigma_{i}, 1 \leq i \leq n$, are arbitrarily fixed, we simply assume that all the upper endpoints of $W_{1}, \ldots, W_{n}$ equal to 1 without loss of generality. We first verify that $X_{n: n} \in \operatorname{GMDA}(h)$ for some $h(\cdot)$. For any $1 \leq i \neq j \leq n$, assume without loss of generality that $\sigma_{i} \geq \sigma_{j}$. It is clear for positive $x$ that

$$
\begin{aligned}
\frac{\mathbb{P}\left(X_{i}>x, X_{j}>x\right)}{\mathbb{P}\left(X_{n: n}>x\right)} & \leq \frac{\mathbb{P}\left(W_{i} Y_{i}>\log x, W_{j} Y_{j}>\log x\right)}{\mathbb{P}\left(W_{i} Y_{i}>\log x\right)} \\
& \leq \frac{\mathbb{P}\left(Y_{i}+Y_{j}>2 \log x\right)}{\mathbb{P}\left(W_{i} Y_{i}>\log x\right)} \\
& \leq \bar{\Phi}\left(\frac{2}{\sqrt{2\left(1+\rho_{i j}\right)}} \frac{\log x-\left(\mu_{i}+\mu_{j}\right) / 2}{\sigma_{i}}\right) / \mathbb{P}\left(W_{i} Y_{i}>\log x\right),
\end{aligned}
$$

where $\Phi(\cdot)$ is the standard normal distribution function. It follows from Lemma A.3 of Tang and Tsitsiashvili (2004) that, for any $w \in(0,1)$,

$$
\mathbb{P}\left(W_{i} Y_{i}>x\right) \sim \mathbb{P}\left(W_{i} Y_{i}>x, W_{i}>w\right) \geq \mathbb{P}\left(Y_{i}>\frac{x}{w}\right) \mathbb{P}\left(W_{i}>w\right)=\bar{\Phi}\left(\frac{x-w \mu_{i}}{w \sigma_{i}}\right) \mathbb{P}\left(W_{i}>w\right) .
$$

Combining the above estimates and choosing $w>\sqrt{2\left(1+\rho_{i j}\right)} / 2$ lead to (4.1), which implies that

$$
\mathbb{P}\left(X_{n: n}>x\right) \sim \sum_{i=1}^{n} \mathbb{P}\left(X_{i}>x\right) \sim \sum_{i \in \Lambda} \mathbb{P}\left(X_{i}>x\right),
$$

where

$$
\Lambda=\left\{i: \sigma_{i}=\sigma=\max _{1 \leq j \leq n} \sigma_{j}, \mu_{i}=\mu=\max _{j: \sigma_{j}=\sigma} \mu_{j}\right\}
$$

and in the last step of (4.5) we used relation (4.4) again. In view of Theorem 1.1 of Hashorva and Weng (2014) (or the last sentence in the first paragraph of their Section 2), $X_{i} \in \operatorname{GMDA}(h)$ for $i \in \Lambda$ with the common auxiliary function $h(\cdot)$ given by

$$
h(x)=\frac{\sigma^{2} x}{\log x-\mu} .
$$

Hence, by Lemma 4.1(a), $X_{n: n} \in \operatorname{GMDA}(h)$ with the same $h(\cdot)$ as above. Then, using the similar procedures as in Example 3.5 of Mitra and Resnick (2009), we can verify (3.1) and (3.2) with such $h(\cdot)$. This establishes the validity of Assumption A.

For $\left(X_{1}, \ldots, X_{n}\right)$ following a multivariate lognormal distribution as in Example 4.1 with $W_{i} \equiv 1$ for $1 \leq i \leq n$, Asmussen and Rojas-Nandayapa (2008) gave in their Theorem 1 a precise asymptotic expansion for $\mathbb{P}\left(\sum_{i=1}^{n} X_{i}>x\right)$; see Hashorva (2013) for some generalizations. Clearly, Example 4.1 indicates that their result is an immediate consequence of our Theorem 3.1.

Example 4.2. Consider the real-valued random variables $X_{1}, \ldots, X_{n}$ with distribution functions $F_{1} \in \mathcal{R}_{-\alpha}, \ldots, F_{n} \in \mathcal{R}_{-\alpha}$ for some $\alpha>0$. Impose on $\left(X_{1}, \ldots, X_{n}\right)$ a multivariate Farlie-GumbelMorgenstern copula (see, e.g., Hashorva and Hüsler (1999)), which implies

$$
\mathbb{P}\left(X_{1} \leq x_{1}, \ldots, X_{n} \leq x_{n}\right)=\prod_{i=1}^{n} F_{i}\left(x_{i}\right)\left(1+\sum_{k=2}^{n} \sum_{1 \leq j_{1}<\cdots<j_{k} \leq n} \theta_{j_{1} \cdots j_{k}} \bar{F}_{j_{1}}\left(x_{j_{1}}\right) \cdots \bar{F}_{j_{k}}\left(x_{j_{k}}\right)\right),
$$


where $\left|\theta_{j_{1} \cdots j_{k}}\right| \leq 1$ are some real numbers such that the right-hand side of (4.7) is a proper multivariate distribution function. We verify Assumptions $\mathbf{B}-\mathbf{C}$ for $X_{1}, \ldots, X_{n}$.

In this case, it is known that, for any $1 \leq i \neq j \leq n$,

$$
\mathbb{P}\left(X_{i}>x_{i}, X_{j}>x_{j}\right)=\bar{F}_{i}\left(x_{i}\right) \bar{F}_{j}\left(x_{j}\right)\left(1+\theta_{i j} F_{i}\left(x_{i}\right) F_{j}\left(x_{j}\right)\right) .
$$

Relation (4.8) obviously implies (4.1). Hence, by Lemma 4.1(b), $X_{n: n} \in \mathcal{R}_{-\alpha} \subset \mathcal{L} \cap \mathcal{D} \subset \mathcal{L}$. Let $h(x)=x^{p} \in \mathcal{H}_{X_{n: n}}$ for some $p \in(1 / 2,1)$. Clearly, $h(\cdot)$ is dominatedly varying and (3.1) holds in view of (4.8). To obtain (3.2), for every $1 \leq i \leq n$ we write $\bar{F}_{i}(x) \sim l_{i}(x) x^{-\alpha}$ with some slowly varying function $l_{i}(\cdot)$. Relation (4.8) gives that, for any $1 \leq i \neq j \leq n$,

$$
\mathbb{P}\left(X_{i}>h(x), X_{j}>h(x)\right) \sim l_{i}\left(x^{p}\right) l_{j}\left(x^{p}\right) x^{-2 p \alpha}\left(1+\theta_{i j}\right)=o\left(\bar{F}_{i}(x)\right)=o(1) \mathbb{P}\left(X_{n: n}>x\right) .
$$

Hence, both Assumptions B and $\mathbf{C}$ hold.

Next, we present an application of our Theorem 3.1 in risk theory. Let $X_{1}, \ldots, X_{n}$ be $n$ insurance risks (claims), which are naturally nonnegative. One of popular risk measures based on the conditional tail expectation (CTE) is defined as (recall $S_{n}=\sum_{i=1}^{n} X_{i}$ )

$$
\mathbb{E}\left(X_{i} \mid S_{n}>\operatorname{VaR}_{q}\left(S_{n}\right)\right), \quad 1 \leq i \leq n,
$$

where $q \in(0,1)$ and $\operatorname{VaR}_{q}\left(S_{n}\right)=\inf \left\{x: \mathbb{P}\left(S_{n} \leq x\right) \geq q\right\}$.

The recent contribution Asimit et al. (2011) and Zhu and Li (2012) proposed to study the asymptotic behaviour of (4.9) as $q \rightarrow 1$ (equivalently, $\operatorname{VaR}_{q}\left(S_{n}\right) \rightarrow \infty$ ).

We consider a slightly broader risk measure defined by

$$
\mathbb{E}\left(\sum_{i \in \Omega} X_{i} \mid S_{n}>\operatorname{VaR}_{q}\left(S_{n}\right)\right)=\sum_{i \in \Omega} \mathbb{E}\left(X_{i} \mid S_{n}>\operatorname{VaR}_{q}\left(S_{n}\right)\right), \quad \varnothing \neq \Omega \subset\{1, \ldots, n\} .
$$

The main motivation for (4.10) is that risks are usually grouped and, for risk management purposes, it is important to calculate the CTE for a group of risks. Applying our Theorem 3.1, we can obtain a pair of asymptotic lower and upper bounds for (4.10) as $q \rightarrow 1$ under Assumption A, which extends Theorem 3.3 of Asimit et al. (2011); see Remark 4.1 below.

Theorem 4.1. Let $X_{1}, \ldots, X_{n}$ be $n$ nonnegative random variables satisfying Assumption $\mathbf{A}$. For every $\varnothing \neq \Omega \subset\{1, \ldots, n\}$, write

$$
0 \leq u=\liminf _{x \rightarrow \infty} \frac{\sum_{i \in \Omega} \mathbb{P}\left(X_{i}>x\right)}{\sum_{i=1}^{n} \mathbb{P}\left(X_{i}>x\right)} \leq \limsup _{x \rightarrow \infty} \frac{\sum_{i \in \Omega} \mathbb{P}\left(X_{i}>x\right)}{\sum_{i=1}^{n} \mathbb{P}\left(X_{i}>x\right)}=U \leq 1 .
$$

Then it holds that

$$
u \leq \liminf _{q \rightarrow 1} \frac{\mathbb{E}\left(\sum_{i \in \Omega} X_{i} \mid S_{n}>\operatorname{VaR}_{q}\left(S_{n}\right)\right)}{\operatorname{VaR}_{q}\left(S_{n}\right)} \leq \limsup _{q \rightarrow 1} \frac{\mathbb{E}\left(\sum_{i \in \Omega} X_{i} \mid S_{n}>\operatorname{VaR}_{q}\left(S_{n}\right)\right)}{\operatorname{VaR}_{q}\left(S_{n}\right)} \leq U .
$$

In view of Lemma 2.4 of Asimit et al. (2011) and Theorem 3.1, we obtain

$$
\operatorname{VaR}_{q}\left(S_{n}\right) \sim \operatorname{VaR}_{q}\left(X_{n: n}\right), \quad q \rightarrow 1,
$$

and hence the relations in (4.11) also hold with the denominator $\operatorname{VaR}_{q}\left(S_{n}\right)$ replaced by $\operatorname{VaR}_{q}\left(X_{n: n}\right)$. Additionally, if we further assume that each $\lim _{x \rightarrow \infty} \mathbb{P}\left(X_{i}>x\right) / \mathbb{P}\left(X_{1}>x\right)$ exists for $1 \leq i \leq n$ like in Assumption 3.3 of Asimit et al. (2011), then our (4.11) with $\Omega \in\{\{i\}: 1 \leq i \leq n\}$ reduces to their precise asymptotic formula (3.30). 
Remark 4.1. In dealing with grouped risks without comparable tails, Theorem 4.1 possesses its own advantages. To see this point, recall the generalized log-normal risks $X_{i}=\mathrm{e}^{W_{i} Y_{i}}$ for $1 \leq i \leq n$ given in Example 4.1. In this case we are naturally concerned with the group of dominating risks, i.e., $\left\{X_{i}: i \in \Lambda\right\}$; see (4.6). Since in general there is no proportional tail-relationship among such dominating risks, Theorem 3.3 of Asimit et al. (2011) cannot be utilized to derive the asymptotics for $\mathbb{E}\left(\sum_{i \in \Lambda} X_{i} \mid S_{n}>\operatorname{VaR}_{q}\left(S_{n}\right)\right)$. However, our Theorem 4.1 and relation (4.5) give that

$$
\mathbb{E}\left(\sum_{i \in \Lambda} X_{i} \mid S_{n}>\operatorname{VaR}_{q}\left(S_{n}\right)\right) \sim \operatorname{VaR}_{q}\left(S_{n}\right), \quad q \rightarrow 1
$$

\section{Proofs}

We state first a lemma and then proceed with the proofs of our main results.

Lemma 5.1. Let $X_{1}, \ldots, X_{n}$ be $n$ real-valued random variables. Assume that the distribution function of $X_{n: n}$ has an infinite upper endpoint.

(a) Relation (3.1) holds if and only if

$$
\left[\frac{X_{k: n}}{h(x)} \mid\left(X_{n: n}>x\right)\right] \stackrel{\mathrm{p}}{\rightarrow} 0, \quad 1 \leq k \leq n-1
$$

where $\stackrel{\mathrm{p}}{\rightarrow}$ " means convergence in probability as $x \rightarrow \infty$.

(b) If further $X_{1}, \ldots, X_{n}$ are mutually independent, then relation (3.1) holds if and only if $h(x) \rightarrow \infty$.

Proof. (a): For the "if" assertion, we use the fact that, for any $1 \leq i \neq j \leq n$ and any $t>0$,

$$
\begin{aligned}
\frac{\mathbb{P}\left(\left|X_{i}\right|>t h(x), X_{j}>x\right)}{\mathbb{P}\left(X_{n: n}>x\right)} & \leq \frac{\mathbb{P}\left(\left|X_{n-1: n}\right|>t h(x), X_{n: n}>x\right)+\mathbb{P}\left(\left|X_{1: n}\right|>t h(x), X_{n: n}>x\right)}{\mathbb{P}\left(X_{n: n}>x\right)} \\
& =\mathbb{P}\left(\frac{\left|X_{n-1: n}\right|}{h(x)}>t \mid X_{n: n}>x\right)+\mathbb{P}\left(\frac{\left|X_{1: n}\right|}{h(x)}>t \mid X_{n: n}>x\right) .
\end{aligned}
$$

For the "only if" assertion, we note that, for any $1 \leq k \leq n-1$ and any $t>0$,

$$
\begin{aligned}
\frac{\mathbb{P}\left(\left|X_{k: n}\right|>t h(x), X_{n: n}>x\right)}{\mathbb{P}\left(X_{n: n}>x\right)} & \leq \frac{\mathbb{P}\left(\bigcup_{1 \leq i \neq j \leq n}\left(\left|X_{i}\right|>t h(x), X_{j}>x\right)\right)}{\mathbb{P}\left(X_{n: n}>x\right)} \\
& \leq \sum_{1 \leq i \neq j \leq n} \frac{\mathbb{P}\left(\left|X_{i}\right|>t h(x), X_{j}>x\right)}{\mathbb{P}\left(X_{n: n}>x\right)} .
\end{aligned}
$$

This completes the proof of assertion (a).

(b): Under the independence condition, it is clear that relation (4.3) holds. Thus, the "if" assertion is obvious. We shall prove the "only if" assertion by contradiction. Therefore, suppose that there exists some $M>0$ and positive numbers $x_{m} \rightarrow \infty$ as $m \rightarrow \infty$ such that $h\left(x_{m}\right) \leq M<\infty$ for all $m$. Since $\left|X_{i}\right|$ does not degenerate at 0 for $1 \leq i \leq n$, we can choose $t$ small enough such that

$$
\rho_{n}=: \min _{1 \leq i \leq n} \mathbb{P}\left(\left|X_{i}\right|>t M\right)>0
$$

Hence, with $X_{n+1}=X_{1}$, we have

$$
\begin{aligned}
\limsup _{x \rightarrow \infty} \sum_{i=1}^{n} \frac{\mathbb{P}\left(\left|X_{i+1}\right|>t h(x)\right) \mathbb{P}\left(X_{i}>x\right)}{\mathbb{P}\left(X_{n: n}>x\right)} & \geq \limsup _{m \rightarrow \infty} \sum_{i=1}^{n} \frac{\mathbb{P}\left(\left|X_{i+1}\right|>t h\left(x_{m}\right)\right) \mathbb{P}\left(X_{i}>x_{m}\right)}{\mathbb{P}\left(X_{n: n}>x_{m}\right)} \\
& \geq \rho_{n} \limsup _{m \rightarrow \infty} \frac{\sum_{i=1}^{n} \mathbb{P}\left(X_{i}>x_{m}\right)}{\mathbb{P}\left(X_{n: n}>x_{m}\right)} \\
& =\rho_{n}>0,
\end{aligned}
$$

which contradicts relation (3.1). 
Proof of Theorem 3.1: Without loss of generality, we only need to prove that, uniformly for $\left(c_{1}, \ldots, c_{n-1}\right) \in[0, d]^{n-1}$,

$$
\mathbb{P}\left(X_{n: n}+\sum_{i=1}^{n-1} c_{i} X_{n-i: n}>x\right) \sim \mathbb{P}\left(X_{n: n}>x\right) \sim \sum_{i=1}^{n} \mathbb{P}\left(X_{i}>x\right) .
$$

The second relation in (5.1) is just relation (4.3), which follows from relations (3.1) (implying relation (4.1)) and (4.2). Hence, the second relation in (5.1) holds under one of Assumptions A-C.

Next, we turn to the first relation in (5.1). For any $t>0$ and the function $h(\cdot)$ specified in Assumption $\mathbf{A}$ or $\mathbf{B}$ or $\mathbf{C}$, we have

$$
\begin{aligned}
\mathbb{P}\left(X_{n: n}+\sum_{i=1}^{n-1} c_{i} X_{n-i: n}>x\right)= & \mathbb{P}\left(X_{n: n}+\sum_{i=1}^{n-1} c_{i} X_{n-i: n}>x, X_{n: n} \leq x-\operatorname{th}(x)\right) \\
& +\mathbb{P}\left(X_{n: n}+\sum_{i=1}^{n-1} c_{i} X_{n-i: n}>x, X_{n: n}>x-\operatorname{th}(x)\right) \\
= & I_{1}(\bar{c}, x)+I_{2}(\bar{c}, x),
\end{aligned}
$$

where $\bar{c} \in[0, d]^{n-1}$ denotes the real vector $\left(c_{1}, \ldots, c_{n-1}\right)$. Recall that under Assumption $\mathbf{A}$ or $\mathbf{B}$ relation (3.2) holds. Thus, in these two cases, we estimate $I_{1}(\bar{c}, x)$ as

$$
I_{1}(\bar{c}, x) \leq \mathbb{P}\left(\sum_{i=1}^{n-1} c_{i} X_{n-i: n}>\operatorname{th}(x)\right) \leq \mathbb{P}\left((n-1) d X_{n-1: n}>t h(x)\right) .
$$

By relation (3.2), for $t>(n-1) d L$, it holds uniformly for $\bar{c} \in[0, d]^{n-1}$ that

$$
\begin{aligned}
I_{1}(\bar{c}, x) & \leq \mathbb{P}\left((n-1) d X_{n-1: n}>\operatorname{th}(x),(n-1) d X_{n: n}>\operatorname{th}(x)\right) \\
& \leq \sum_{1 \leq i \neq j \leq n} \mathbb{P}\left(X_{i}>\operatorname{Lh}(x), X_{j}>\operatorname{Lh}(x)\right) \\
& =o(1) \mathbb{P}\left(X_{n: n}>x\right) .
\end{aligned}
$$

Under Assumption $\mathbf{C}$, we deal with $I_{1}(\bar{c}, x)$ as

$$
\begin{aligned}
I_{1}(\bar{c}, x) & \leq \mathbb{P}\left(X_{n: n}+\sum_{i=1}^{n-1} c_{i} X_{n-i: n}>x, \sum_{i=1}^{n-1} c_{i} X_{n-i: n}>t h(x)\right) \\
& \leq \mathbb{P}\left(n(d+1) X_{n: n}>x,(n-1) d X_{n-1: n}>t h(x)\right) \\
& \leq \sum_{1 \leq i \neq j \leq n} \mathbb{P}\left(X_{i}>\frac{x}{n(d+1)}, X_{j}>\frac{t h(x)}{(n-1) d}\right) \\
& =\sum_{1 \leq i \neq j \leq n} \mathbb{P}\left(X_{i}>\frac{x}{n(d+1)}, X_{j}>\frac{t}{(n-1) d} \frac{h(x)}{h(x / n(d+1))} h\left(\frac{x}{n(d+1)}\right)\right) .
\end{aligned}
$$

Recalling that $h(\cdot)$ is dominatedly varying, there exists some $\delta>0$ such that $h(x) / h(x / n(d+1)) \geq \delta$ for large $x$. Thus, we have

$$
\begin{aligned}
I_{1}(\bar{c}, x) & \leq \sum_{1 \leq i \neq j \leq n} \mathbb{P}\left(X_{i}>\frac{x}{n(d+1)}, X_{j}>\frac{t \delta}{(n-1) d} h\left(\frac{x}{n(d+1)}\right)\right) \\
& =o(1) \mathbb{P}\left(X_{n: n}>\frac{x}{n(d+1)}\right) \\
& =o(1) \mathbb{P}\left(X_{n: n}>x\right),
\end{aligned}
$$

where in the second and the last steps we used (3.1) and $F \in \mathcal{D}$, respectively. Hence, under one of Assumptions A-C, relation (5.2) holds uniformly for $\bar{c} \in[0, d]^{n-1}$. 
For $I_{2}(\bar{c}, x)$, we further write

$$
\begin{aligned}
I_{2}(\bar{c}, x) & =\mathbb{P}\left(X_{n: n}+\sum_{i=1}^{n-1} c_{i} X_{n-i: n}>x \mid X_{n: n}>x-t h(x)\right) \mathbb{P}\left(X_{n: n}>x-t h(x)\right) \\
& =J_{1}(\bar{c}, x) J_{2}(x) .
\end{aligned}
$$

It is clear that

$$
J_{1}(\bar{c}, x)=\mathbb{P}\left(\frac{X_{n: n}-x}{h(x)}+\frac{\sum_{i=1}^{n-1} c_{i} X_{n-i: n}}{h(x)}>0 \mid X_{n: n}>x-t h(x)\right) .
$$

Hence, by Lemma 5.1(a) and property (iii) of $h(\cdot)$, we obtain that, uniformly for $\bar{c} \in[0, d]^{n-1}$,

$$
\left[\frac{\sum_{i=1}^{n-1} c_{i} X_{n-i: n}}{h(x)} \mid\left(X_{n: n}>x-t h(x)\right)\right]=\left[\frac{\sum_{i=1}^{n-1} c_{i} X_{n-i: n}}{h(x-t h(x))} \frac{h(x-t h(x))}{h(x)} \mid\left(X_{n: n}>x-t h(x)\right)\right] \stackrel{\mathrm{p}}{\rightarrow} 0 .
$$

Additionally, under Assumption A with $h(\cdot)$ satisfying property (iii'), we can derive that

$$
\begin{aligned}
& {\left[\frac{X_{n: n}-x}{h(x)} \mid\left(X_{n: n}>x-t h(x)\right)\right] } \\
= & {\left[\left(\frac{X_{n: n}-(x-t h(x))}{h(x-t h(x))} \frac{h(x-t h(x))}{h(x)}-t\right) \mid\left(X_{n: n}>x-t h(x)\right)\right] \stackrel{\mathrm{d}}{\rightarrow} Y-t, }
\end{aligned}
$$

where " $\stackrel{\text { d }}{\rightarrow}$ " means convergence in distribution as $x \rightarrow \infty$ and $Y$ is an exponential random variable with expectation 1. Note further that relation (5.4) holds with $Y=\infty$ under Assumption B or $\mathbf{C}$, because of $X_{n: n} \in \mathcal{L}$ and property (ii). Hence, it holds uniformly for $\bar{c} \in[0, d]^{n-1}$ that

$$
\left[\left(\frac{X_{n: n}-x}{h(x)}+\frac{\sum_{i=1}^{n-1} c_{i} X_{n-i: n}}{h(x)}\right) \mid\left(X_{n: n}>x-t h(x)\right)\right] \stackrel{\mathrm{d}}{\rightarrow} Y-t
$$

which implies that, uniformly for $\bar{c} \in[0, d]^{n-1}$,

$$
\lim _{x \rightarrow \infty} J_{1}(\bar{c}, x)=\mathbb{P}(Y-t>0)=\left\{\begin{array}{ll}
\mathrm{e}^{-t}, & \text { under Assumption } \mathbf{A} \\
1, & \text { under Assumption B or } \mathbf{C}
\end{array} .\right.
$$

On the other hand, we have

$$
\lim _{x \rightarrow \infty} \frac{J_{2}(x)}{\mathbb{P}\left(X_{n: n}>x\right)}=\left\{\begin{array}{ll}
\mathrm{e}^{t}, & \text { under Assumption } \mathbf{A} \\
1, & \text { under Assumption } \mathbf{B} \text { or } \mathbf{C}
\end{array} .\right.
$$

Plugging (5.5) and (5.6) into (5.3) leads to that the relation

$$
I_{2}(\bar{c}, x) \sim \mathbb{P}\left(X_{n: n}>x\right)
$$

holds uniformly for $\bar{c} \in[0, d]^{n-1}$ under one of Assumptions A-C. This, together with (5.2), completes the proof.

For the proof of Theorem 4.1, we shall need a crucial property of distribution functions in the GMDA referred to as the Davis-Resnick tail property; see Proposition 1.1 of Davis and Resnick (1988) or relation (5) of Balakrishnan and Hashorva (2013). Namely, for any distribution function $F \in \operatorname{GMDA}(h)$ with an infinite upper endpoint, the following bound holds for all large $x$ and $\varepsilon>0$ :

$$
\frac{\bar{F}(x+h(x) s)}{\bar{F}(x)} \leq(1+\varepsilon)(1+\varepsilon s)^{-1 / \varepsilon}, \quad \forall s \geq 0,
$$


where $h(\cdot)$ is a particular scaling function such that for all large $x>x_{0}$ we have $\bar{F}(x)=c(x) \exp \left(-\int_{x_{0}}^{x} h(t) \mathrm{d} t\right)$ with $c(\cdot)$ a measurable function satisfying $\lim _{x \rightarrow \infty} c(x)=c>0$. Note in passing that any other scaling function $h^{*}$ such that $F \in \operatorname{GMDA}\left(h^{*}\right)$ is asymptotically equivalent to $h$.

Proof of Theorem 4.1: Without loss of generality, we only prove the case of $\Omega=\{1, \ldots, m\}$ for some $1 \leq m \leq n$. Following the proof of Theorem 3.3 of Asimit et al. (2011), we write

$$
\begin{aligned}
\mathbb{E}\left(S_{m} \mid S_{n}>x\right) & =\left(\int_{0}^{h(x)}+\int_{h(x)}^{x}+\int_{x}^{\infty}\right) \mathbb{P}\left(S_{m}>z \mid S_{n}>x\right) \mathrm{d} z \\
& =I_{1}(x)+I_{2}(x)+I_{3}(x) .
\end{aligned}
$$

It is clear that $I_{1}(x) \leq h(x)=o(x)$. By the change of variable $z=x+h(x) s$, we have

$$
\begin{aligned}
I_{3}(x) & =h(x) \int_{0}^{\infty} \mathbb{P}\left(S_{m}>x+h(x) s \mid S_{n}>x\right) \mathrm{d} s \\
& \leq h(x) \int_{0}^{\infty} \frac{\mathbb{P}\left(S_{n}>x+h(x) s\right)}{\mathbb{P}\left(S_{n}>x\right)} \mathrm{d} s \\
& \sim h(x) \int_{0}^{\infty} \frac{\mathbb{P}\left(X_{n: n}>x+h(x) s\right)}{\mathbb{P}\left(X_{n: n}>x\right)} \mathrm{d} s,
\end{aligned}
$$

where in the last step we used Theorem 3.1. Hence, by the aforementioned Davis-Resnick tail property (5.7) for $X_{n: n} \in \operatorname{GMDA}(h)$ and the Dominated Convergence Theorem, we have

$$
I_{3}(x) \leq(1+o(1)) h(x) \int_{0}^{\infty} \mathrm{e}^{-s} \mathrm{~d} s=o(x) .
$$

It remains to verify that

$$
\liminf _{x \rightarrow \infty} \frac{\sum_{i=1}^{m} \mathbb{P}\left(X_{i}>x\right)}{\sum_{i=1}^{n} \mathbb{P}\left(X_{i}>x\right)} \leq \liminf _{x \rightarrow \infty} \frac{I_{2}(x)}{x} \leq \limsup _{x \rightarrow \infty} \frac{I_{2}(x)}{x} \leq \limsup _{x \rightarrow \infty} \frac{\sum_{i=1}^{m} \mathbb{P}\left(X_{i}>x\right)}{\sum_{i=1}^{n} \mathbb{P}\left(X_{i}>x\right)} .
$$

To this purpose, we further write

$$
\begin{aligned}
\liminf _{x \rightarrow \infty} \frac{I_{2}(x)}{x} & \geq \liminf _{x \rightarrow \infty} \frac{(x-h(x)) \mathbb{P}\left(S_{m}>x \mid S_{n}>x\right)}{x} \\
& =\liminf _{x \rightarrow \infty} \frac{\mathbb{P}\left(S_{m}>x\right)}{\mathbb{P}\left(S_{n}>x\right)}-\lim _{x \rightarrow \infty} \frac{h(x)}{x} \mathbb{P}\left(S_{m}>x \mid S_{n}>x\right) \\
& \geq \liminf _{x \rightarrow \infty} \frac{\sum_{i=1}^{m} \mathbb{P}\left(X_{i}>x\right)-\sum_{1 \leq i<j \leq m} \mathbb{P}\left(X_{i}>x, X_{j}>x\right)}{\mathbb{P}\left(S_{n}>x\right)} \\
& =\liminf _{x \rightarrow \infty} \frac{\sum_{i=1}^{m} \mathbb{P}\left(X_{i}>x\right)}{\sum_{i=1}^{n} \mathbb{P}\left(X_{i}>x\right)}
\end{aligned}
$$

where in the last step we used Theorem 3.1 and Assumption A. Additionally, it holds that

$$
\begin{aligned}
& \mathbb{P}\left(S_{m}>h(x), S_{n}>x\right) \\
= & \mathbb{P}\left(S_{n}>x\right)-\mathbb{P}\left(S_{m} \leq h(x), S_{n}>x\right) \\
\leq & \mathbb{P}\left(S_{n}>x\right)-\mathbb{P}\left(S_{m} \leq h(x), \bigcup_{j=m+1}^{n}\left(X_{j}>x\right)\right) \\
= & \mathbb{P}\left(S_{n}>x\right)-\mathbb{P}\left(\bigcup_{j=m+1}^{n}\left(X_{j}>x\right)\right)+\mathbb{P}\left(S_{m}>h(x), \bigcup_{j=m+1}^{n}\left(X_{j}>x\right)\right) \\
\leq & \mathbb{P}\left(S_{n}>x\right)-\sum_{j=m+1}^{n} \mathbb{P}\left(X_{j}>x\right)+\sum_{m+1 \leq j<k \leq n} \mathbb{P}\left(X_{j}>x, X_{k}>x\right)+\sum_{1 \leq i \leq m<j \leq n} \mathbb{P}\left(X_{i}>\frac{h(x)}{m}, X_{j}>x\right) .
\end{aligned}
$$


It follows from Theorem 3.1 and Assumption $\mathbf{A}$ that the last two terms in the above relation are asymptotically negligible compared with $\mathbb{P}\left(S_{n}>x\right)$. Hence, by Theorem 3.1 again, we have

$$
\begin{aligned}
\limsup _{x \rightarrow \infty} \frac{I_{2}(x)}{x} & \leq \limsup _{x \rightarrow \infty} \frac{\mathbb{P}\left(S_{m}>h(x), S_{n}>x\right)}{\mathbb{P}\left(S_{n}>x\right)} \\
& \leq \limsup _{x \rightarrow \infty} \frac{\mathbb{P}\left(S_{n}>x\right)-\sum_{j=m+1}^{n} \mathbb{P}\left(X_{j}>x\right)}{\mathbb{P}\left(S_{n}>x\right)} \\
& =\limsup _{x \rightarrow \infty} \frac{\sum_{i=1}^{m} \mathbb{P}\left(X_{i}>x\right)}{\sum_{i=1}^{n} \mathbb{P}\left(X_{i}>x\right)},
\end{aligned}
$$

which together with (5.8) completes the proof.

Acknowledgment. We are very much in debt to the two reviewers and an Editor who carefully read our manuscript and showed us how to significantly improve it. The authors were partially supported by the Swiss National Science Foundation Project 200021-140633/1 and the project RARE -318984 (an FP7 Marie Curie IRSES Fellowship). The second author also acknowledges the support from the National Natural Science Foundation of China (Grant No.: 11201245).

\section{References}

[1] Asimit, V.A.; Hashorva, E.; Kortschak, D. Asymptotic tail probability of randomly weighted large risks. Available at SSRN: http://dx.doi.org/10.2139/ssrn.1993114, (2013).

[2] Asimit, A. V.; Furman, E.; Tang, Q.; Vernic, R. Asymptotics for risk capital allocations based on conditional tail expectation. Insurance Math. Econom. 49 (2011), no. 3, 310-324.

[3] Asmussen, S.; Blanchet, J.; Juneja, S.; Rojas-Nandayapa, L. Efficient simulation of tail probabilities of sums of correlated lognormals. Ann. Oper. Res. 189 (2011), 5-23.

[4] Asmussen, S.; Foss, S. On exceedance times for some processes with dependent increments. J. Appl. Probab. (2014), to appear.

[5] Asmussen, S.; Rojas-Nandayapa, L. Asymptotics of sums of lognormal random variables with Gaussian copula. Statist. Probab. Lett. 78 (2008), no. 16, 2709-2714.

[6] Balakrishnan, N.; Hashorva, E. Scale mixtures of Kotz-Dirichlet distributions. J. Multivariate Anal. 113 (2013), 48-58.

[7] Cai, J.; Tang, Q. On max-sum equivalence and convolution closure of heavy-tailed distributions and their applications. J. Appl. Probab. 41 (2004), no. 1, 117-130.

[8] Davis, R.; Resnick, S. I. Extremes of moving averages of random variables from the domain of attraction of the double exponential distribution. Stochastic Process. Appl. 30 (1988), no. 1, 4168.

[9] Embrechts, P.; Hashorva, E.; Mikosch, T. Aggregation of log-linear risks. J. Appl. Probab., (2014), in press.

[10] Embrechts, P.; Goldie, C. M. On closure and factorization properties of subexponential and related distributions. J. Austral. Math. Soc. Ser. A 29 (1980), no. 2, 243-256. 
[11] Embrechts, P.; Klüppelberg, C.; Mikosch, T. Modelling Extremal Events. Springer-Verlag, Berlin, 1997.

[12] Foss, S.; Konstantopoulos, T.; Zachary, S. Discrete and continuous time modulated random walks with heavy-tailed increments. J. Theoret. Probab. 20 (2007), no. 3, 581-612.

[13] Foss, S.; Richards, A. On sums of conditionally independent subexponential random variables. Math. Oper. Res. 35 (2010), 102-119.

[14] Foss, S.; Korshunov, D.; Zachary, S. An Introduction to Heavy-tailed and Subexponential Distributions. 2nd Edition. Springer-Verlag, New York, 2013.

[15] Geluk, J. Some closure properties for subexponential distributions. Statist. Probab. Lett. 79 (2009), no. $8,1108-1111$.

[16] Goldie, C. M.; Resnick, S. Distributions that are both subexponential and in the domain of attraction of an extreme-value distribution. Adv. in Appl. Probab. 20 (1988), no. 4, 706-718.

[17] Gulisashvili, A.; Tankov, P. Tail behavior of sums and differences of log-normal random variables. Available at www.arXiv:1309.3057, (2013).

[18] Hashorva, E. Exact tail asymptotics of aggregated parametrised risk. J. Math. Anal. Appl. 400 (2013), no. 1, 187-199.

[19] Hashorva, E.; Weng, Z. Limit laws for maxima of contracted stationary Gaussian sequences. Comm. Stat. Theory Meth. (2014), to appear.

[20] Hashorva, E.; Hüsler, J. Extreme values in FGM random sequences. J. Multivariate Anal. 68 (1999), no. 2, 212-225.

[21] Kortschak, D.; Hashorva, E. Efficient simulation of tail probabilities for sums of log-elliptical risks. J. Comp. Appl. Math. 247 (2013), 53-67.

[22] Li, J.; Tang, Q. A note on max-sum equivalence. Statist. Probab. Lett. 80 (2010), no. 23-24, 17201723.

[23] Li, J.; Tang, Q.; Wu, R. Subexponential tails of discounted aggregate claims in a time-dependent renewal risk model. Adv. in Appl. Probab. 42 (2010), no. 4, 1126-1146.

[24] Mitra, A.; Resnick, S. I. Aggregation of rapidly varying risks and asymptotic independence. Adv. in Appl. Probab. 41 (2009), no. 3, 797-828.

[25] Resnick, S. I. Extreme Values, Regular Variation, and Point Processes. Springer-Verlag, New York, 1987.

[26] Tang, Q.; Tsitsiashvili, G. Finite- and infinite-time ruin probabilities in the presence of stochastic returns on investments. Adv. in Appl. Probab. 36 (2004), no. 4, 1278-1299.

[27] Yang, Y. On extremal behaviour of aggregation of largest claims. Preprint, (2014).

[28] Zhu, L.; Li, H. Asymptotic analysis of multivariate tail conditional expectations. N. Am. Actuar. J. 16 (2012), no. 3, 350-363. 